Revue des patrimoines

\title{
Patrimoine technique méconnu des mines d'Alsace et de Moselle : les machines d'extraction
}

\section{Chip Buchheit}

\section{OpenEdition}

\section{Journals}

Édition électronique

URL : http://journals.openedition.org/insitu/2869

DOI : 10.4000/insitu.2869

ISSN : $1630-7305$

\section{Éditeur}

Ministère de la culture

Référence électronique

Chip Buchheit, «Patrimoine technique méconnu des mines d'Alsace et de Moselle : les machines d'extraction », In Situ [En ligne], $8 \mid$ 2007, mis en ligne le 01 mars 2007, consulté le 01 mai 2019. URL http://journals.openedition.org/insitu/2869; DOI : 10.4000/insitu.2869

Ce document a été généré automatiquement le 1 mai 2019.

\section{(c) $($ i) $(9)$}

In Situ Revues des patrimoines est mis à disposition selon les termes de la licence Creative Commons Attribution - Pas d'Utilisation Commerciale - Pas de Modification 4.0 International. 


\title{
Patrimoine technique méconnu des mines d'Alsace et de Moselle : les machines d'extraction
}

\author{
Chip Buchheit
}

1 Cet article fait suite à plusieurs études sur le patrimoine minier du bassin houiller lorrain (Moselle) et des mines de potasse d'Alsace (MDPA, Haut-Rhin). Le champ des investigations, élargi à l'occasion aux problématiques économiques, industrielles ou socio-techniques, a toujours nécessité de recenser les installations et d'évaluer leur intérêt patrimonial. La méthodologie de l'Inventaire a beaucoup servi, même si elle s'est souvent combinée à d'autres outils pour mieux répondre aux attentes des commanditaires.

2 Les techniques minières y sont invariablement apparues comme particulièrement intéressantes car elles répondent aux exigences d'un milieu qui compte parmi les plus contraignants qui soient. Elles le sont aussi parce que leur modernisation a largement contribué au progrès technique général. Faut-il rappeler que c'est pour répondre aux besoins des exploitants miniers qu'ont été mises au point les inventions capitales que sont la double voie de roulage et la machine à vapeur? Sans son application à la remontée $\mathrm{du}$ charbon, les modes d'exploitation dits "de surface" ${ }^{1}$ n'auraient pu être avantageusement remplacés, dès le dernier quart du XVIIIe siècle, par les "puits verticaux ", et des gisements jusque-là inaccessibles n'auraient pu être progressivement exploités. Le phénomène même de l'industrialisation en eût été sérieusement compromis.

Les machines d'extraction figurent elles aussi au rang des inventions majeures mises au service du formidable essor de l'industrie minière des XIXe et XXe siècles (fig. $\mathbf{n}^{\circ} \mathbf{1}$ ). 
Figure 1

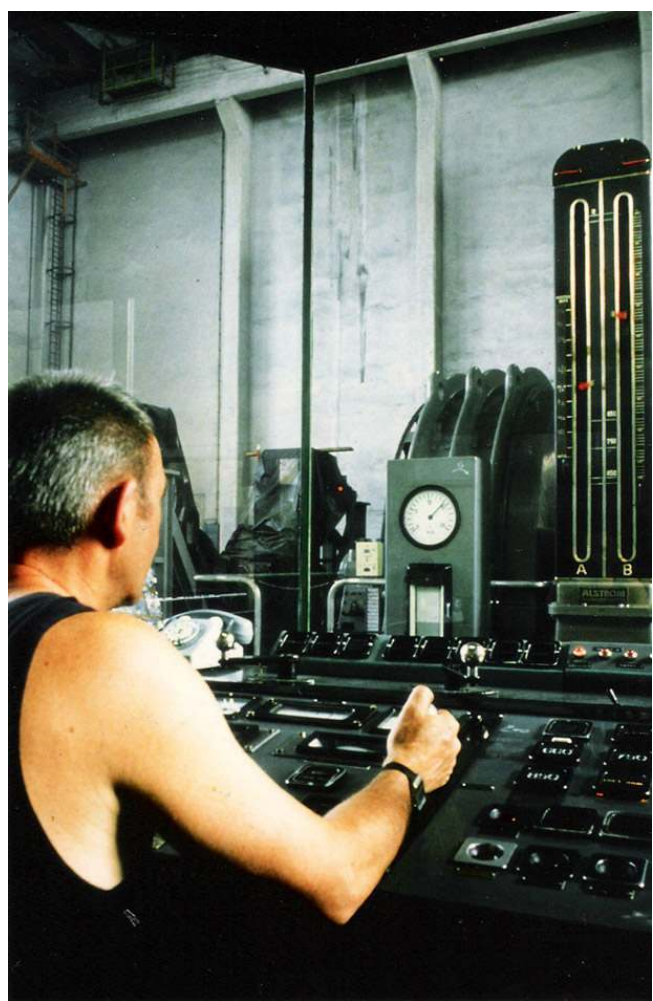

Machiniste à la manœuvre en 1983 au puits Wendel 3 (Petite-Rosselle). CCSTI-Musée du bassin houiller lorrain

(c) CCSTI-Musée du bassin houiller lorrain, 1983

Installées au sol, dans une salle des machines voisine d'un chevalement ou bien juchées
en tête d'une tour d'extraction, elles constituent des objets techniques d'exception
capables de révéler la configuration et la puissance des exploitations.

5 Avec le déclin de l'industrie extractive en France, perçu comme irréversible depuis les années 1970, l'équipement des mines est entré à son tour dans le champ du patrimoine, les différents bassins ayant été l'objet, à partir de 1986, d'un recensement de leurs installations de surface. C'est bien autour des puits, passages obligés entre le fond et le jour, que se retrouvent les structures les plus caractéristiques de l'industrie extractive : chevalement ou tour d'extraction, recette et salle des machines d'extraction. Pour bien comprendre les particularités de ce type d'équipement, il n'est pas inutile de procéder à une brève mise en perspective historique et technique.

\section{Les machines d'extraction, objets techniques par excellence}

Des mécanismes élévateurs de charges 
Figure 2

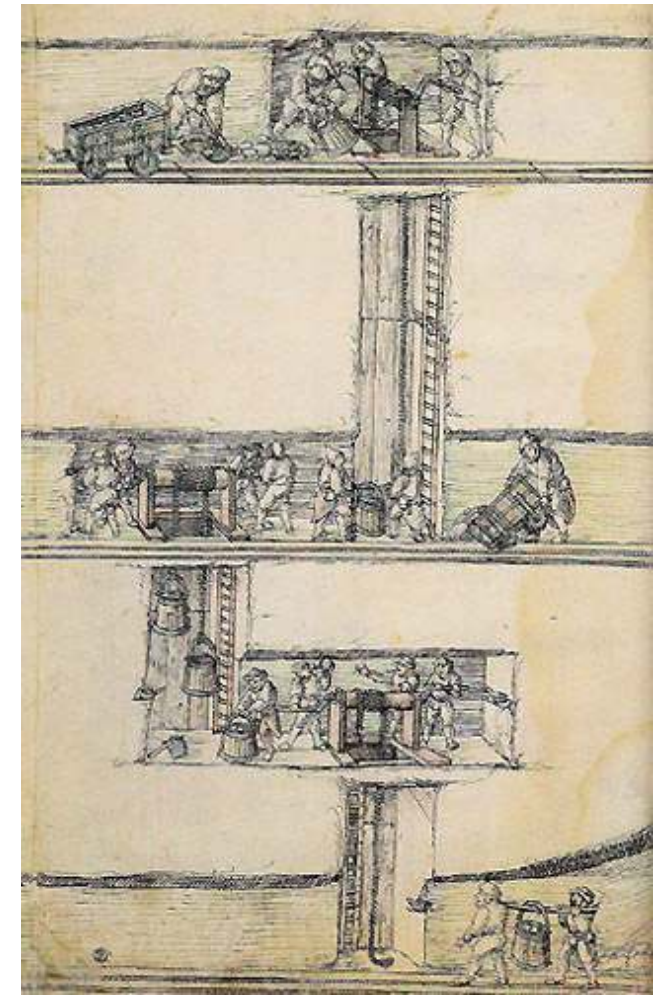

Coupe d'une mine au début du XVIe siècle, publiée dans La Mine mode d'emploi. La Rouge Myne de Sainct Nicolas de La Croix dessinée par Heinrich Groff, présenté par E. Brugerolles, H. Bari, P. Benoît, P. Fluck et H. Schoen. Paris : Découvertes Gallimard Albums, 1992.

Pour les hommes de l'art, l'extraction minière recouvre l'ensemble des opérations assurant le transport du minerai, depuis le front de taille où il est abattu jusqu'au jour (fig. $\mathbf{n}^{\circ}$ 2). Pour autant, les engins et machineries progressivement établis pour élever les produits n'interviennent qu'aux endroits où ils ne peuvent être ni portés, ni tirés, ni roulés, au pied des "puits droits" et des puits inclinés creusés dans la roche et débouchant à la surface.

\section{Apparition d'une nouvelle filière technique}

7 Cette fonction ponctuelle d'élévation fut longtemps servie par des engins présentant les caractères rudimentaires du treuil de levage (également utilisé, à l'occasion, pour extraire l'eau), puis du baritel à cheval (ou manège) (fig. $\mathbf{n}^{\circ} 3$ ) et de la roue hydraulique qui en démultiplièrent les capacités. 
Figure 3

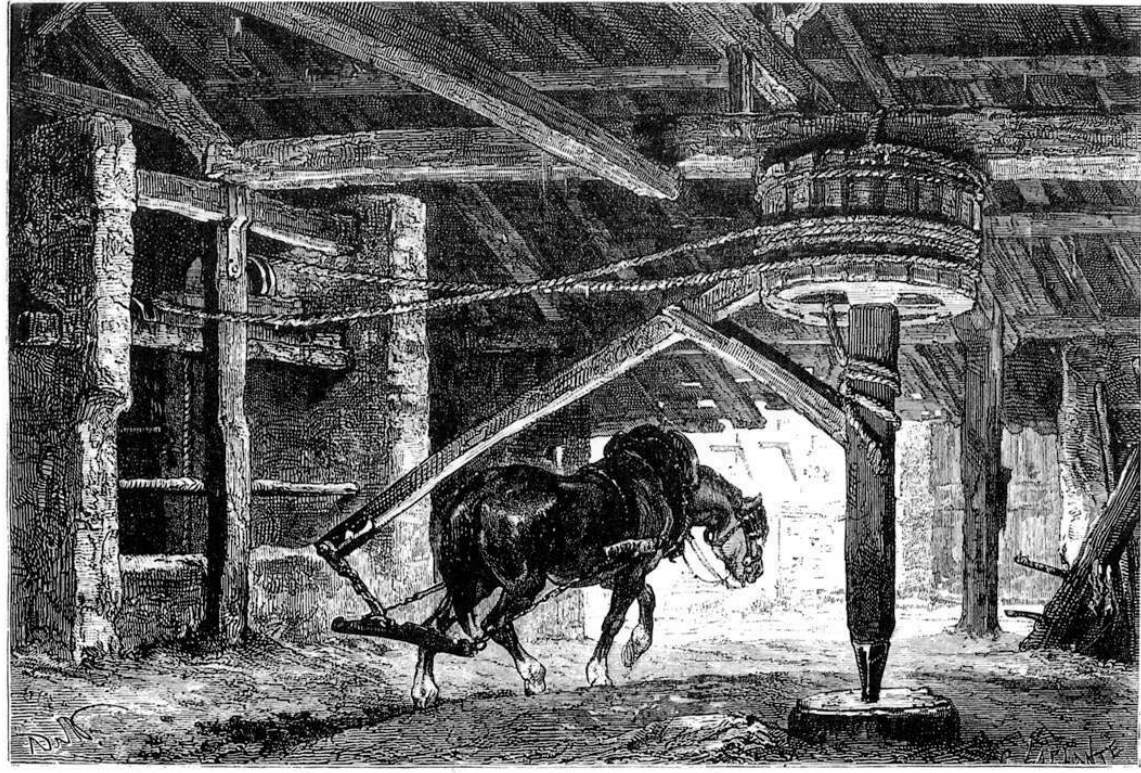

Fig. 59. - L'antiạue baritel ou manége des houillères, daprès Lançon

L'antique baritel ou manège des houillères, d'après Lançon. Manège actionné par un cheval. Ce type d'équipement a été utilisé jusqu'au milieu du XIXe siècle. Publié dans : SIMONIN, Louis. LA VIE

SOUTERRAINE. LeS MiNes et Les MineURS, réédition de 1867. Coll. Milieux. Seyssel : Champ Vallon, 1982

8 Avec l'avènement de nouvelles énergies motrices (vapeur puis électricité), on vit apparaitre de nouveaux engins de levage dont les mécanismes, bien plus perfectionnés, signalent l'apparition sur les carreaux de mines d'une nouvelle filière technique d'abord appelée "machine de rotation" - car inspirée du système Cartwright - puis "machine d'extraction".

Figure 4

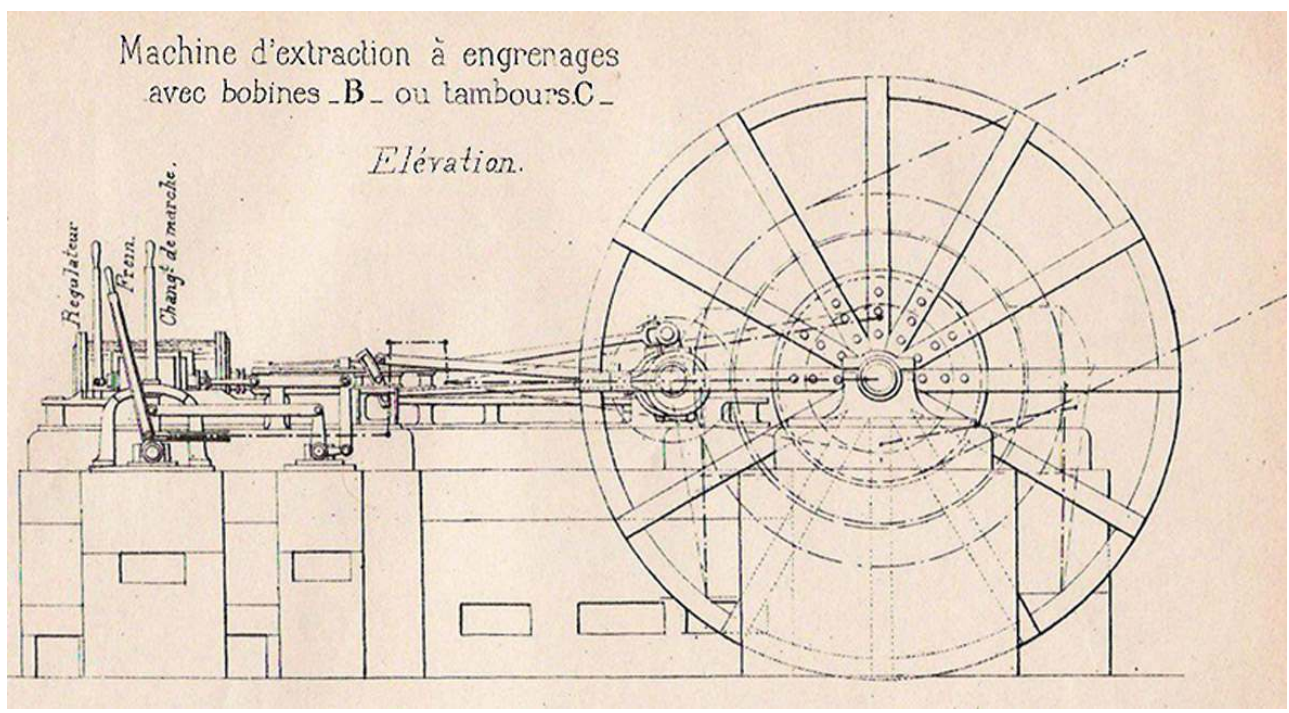

Machine d'extraction à bobine courante à la fin du XIXe siècle (publiée dans WURGLER. EXPLOITATION DES MINES, 1887-1888, pl. LXXXVI). 
9 des treuils continuent d'être employés dans les chantiers souterrains, où ils gagnent en puissance mais sans connaître de modifications significatives, les machines d'extraction sont devenues complexes, objets de continuels perfectionnements (fig. $\mathbf{n}^{\circ} \mathbf{4}$ ). De leurs capacités bien calibrées dépendait alors la bonne marche de l'ensemble des exploitations.

On résume ci-dessous l'évolution de ces engins de levage (tableau A).

Tableau A : évolution morpho-technique des équipements d'extraction

\begin{tabular}{|l|c|c|c|}
\hline \multicolumn{1}{|c|}{ moteur(s) } & engins & périodes \\
\hline dispositif archaïque & bras de l'homme & treuil & Antiquité/XIXe s \\
\hline $\begin{array}{l}\text { dispositif } \\
\text { traditionnel }\end{array}$ & cheval & manège & \\
\hline dispositif classique & vapeur & roue ou turbine & XVIIIe/XIXe s. \\
& & $\begin{array}{c}\text { tambour } \\
\text { bobine } \\
\text { poulie }\end{array}$ & $\begin{array}{c}\text { XIXe/XXe s. } \\
(1800-m i \text { XXe s.) }\end{array}$ \\
\hline $\begin{array}{l}\text { dispositif } \\
\text { technologique }\end{array}$ & électricité & & $\begin{array}{c}\text { XXe } \mathrm{s} . / \mathrm{XXI} \mathrm{s} . \\
\text { (à partir de 1902) }\end{array}$ \\
\hline
\end{tabular}

1

En France, ces machines d'un nouveau type fonctionnant à la vapeur sont apparues pour la première fois en 1800 dans les houillères de Littry (Calvados). Elles sont sorties des ateliers des Frères Périer, à Chaillot (Paris, 16 $\left.{ }^{\mathrm{e}}\right)^{2}$. Bientôt imitée, l'invention gagna rapidement les houillères du nord de la France (les mines d'Anzin en possèdent déjà 21 en 1810) avant de se répandre progressivement dans les autres bassins. Le tout premier puits $\mathrm{du}$ bassin houiller lorrain - le puits de Schoeneck - en est équipé dès 1835 (machine Saulnier développant 8 chevaux) (fig. $n^{\circ} 5$ ). 
Figure 5

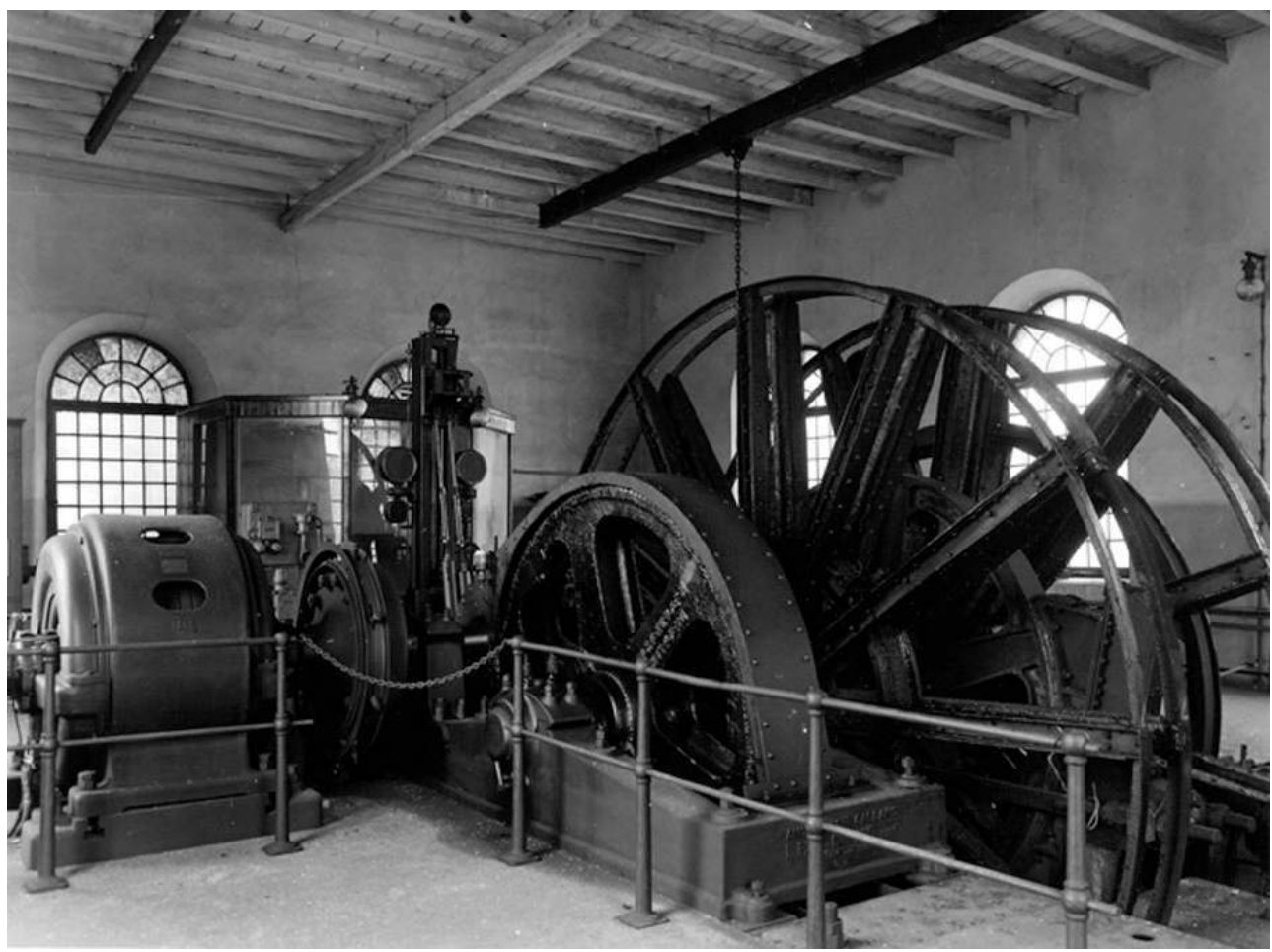

Machine d'extraction à bobine du puits Joseph (Petite-Rosselle), établie en 1898 et disparue en 1967. Coll. Houillères du Bassin de Lorraine - Service audiovisuel

(c) Houillère du Bassin de Lorraine.

12 Il faut savoir que les équipements établis en Moselle (bassin houiller exploité entre 1821 et 2005) et en Alsace (mines de potasse en activité entre 1910 et 2004) présentent un intérêt particulier du fait du demi-siècle que dura l'annexion allemande (1870-1918), car s'y retrouve la " compétition " à laquelle se sont livrés, entre 1902 et 1950, les tenants de l'extraction à vapeur et les promoteurs de l'extraction électrique ${ }^{3}$. Les mines de l'ancien Reichsland d'Alsace-Moselle y ont tour à tour connu l'engouement initial des exploitants allemands pour les machines électriques, entre 1902 et 1918, avant de partager l'option “ tout électrique " adoptée par les exploitants français au lendemain de l'Armistice". 


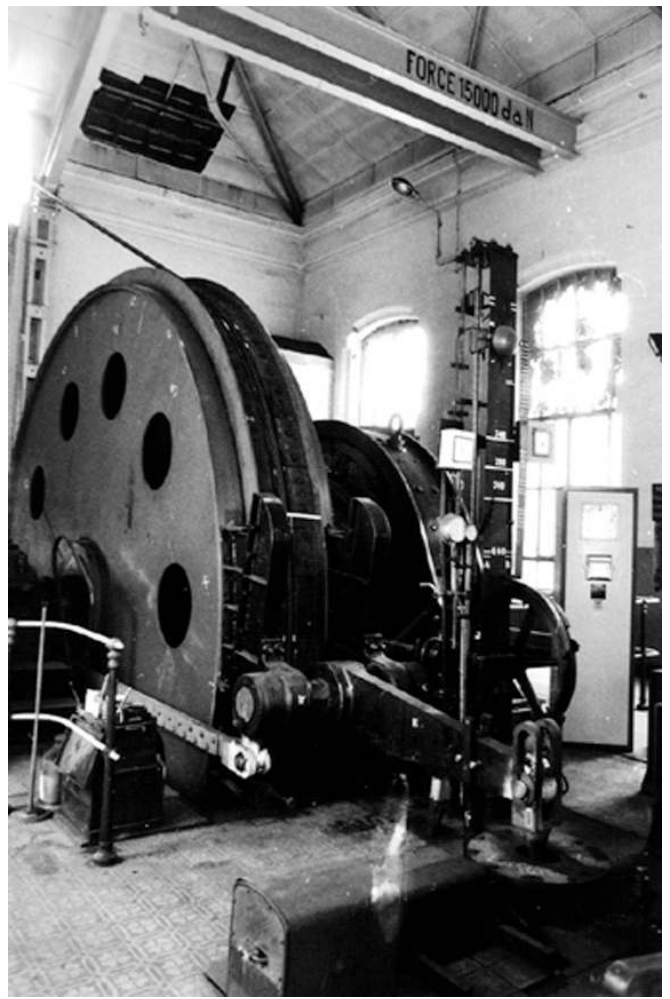

Machine d'extraction du puits Simon 1, établie en 1909 à Forbach. Buchheit, Chip

(c) Buchheit, Chip, 1997.

Cette alternance des souverainetés explique pourquoi les machines d'extraction électriques les plus anciennes de France se retrouvent aujourd'hui en Moselle et en Alsace, sur le carreau des puits Simon 1 et 2 (fig. $\mathbf{n}^{\circ}$ 6), à Forbach (machines d'extraction datant de 1908 et 1913) et au puits Rodolphe 1 de Pulversheim (machine de 1913). Ces équipements doivent être regardés comme un héritage - précieux - des années allemandes.

\section{Les machines d'extraction : objet technique à part entière ou élément d'un système plus complexe?}

Les machines d'extraction constituent, a priori, des objets techniques à part entière, composées d'un bâti, d'un moteur, d'un organe d'enroulement guidant le ou les câbles, d'un poste de conduite assurant l'inversion de la marche, de puissants dispositifs de freinage et de plusieurs instruments contrôlant les cordées. Mais à la différence des treuils, elles ne peuvent fonctionner sans les autres importants équipements que sont les chevalements (ou les tours d'extraction) et les recettes qui abritent les armements des puits, le tout formant un système technique fonctionnellement indissociable (fig. $\mathbf{n}^{\circ} \mathbf{7}$ ). 
Figure 7

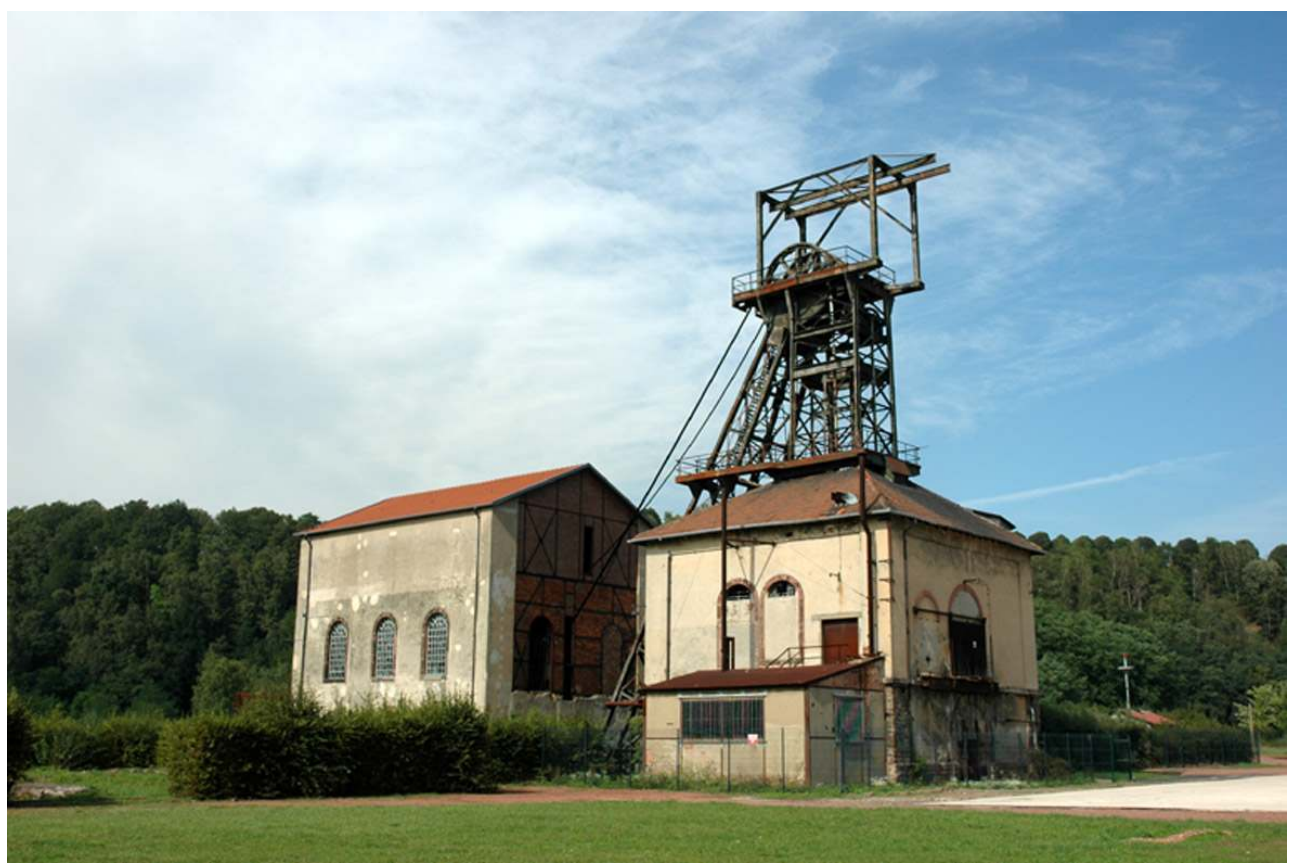

Un système d'extraction complet au puits Vuillemin 2 datant de 1884-1885 à Petite-Rosselle,

comprenant une recette surmontée d'un chevalement et le bâtiment abritant la machine d'extraction. Buchheit, Chip

(c) Buchheit, Chip, 2003.

Il devient dès lors bien difficile d'appréhender l'un ou l'autre de ces éléments de manière isolée, d'autant plus qu'ils ont subi les mêmes aléas: lors d'un approfondissement de puits, par exemple, chevalement, recette et machine se trouvaient renforcés ou remplacés de concert. Cette simultanéité des renouvellements concerne essentiellement, et dans tous les bassins, les puits d'extraction également appelés "puits principaux". Pour les puits de service, ou " puits secondaires", les modifications ont essentiellement visé les machines dans le cadre d'un renouvellement périodique des équipements fatigués.

16 L'évolution de la filière, depuis 1800 , traduit tout à la fois une montée en puissance formidable des équipements et une diversification de tous les éléments composant la machine. Ainsi, aux gros treuils à tambour sur lesquels s'enroulait autrefois une grosse corde de chanvre se substituèrent successivement les bobines guidant un câble plat (milieu du XIXe siècle), les poulies Koepe “à câble rond d'équilibre " (à partir de 1878)6, puis les tambours bicylindroconiques (entre les deux guerres) (fig. $\left.\mathbf{n}^{\circ} \mathbf{8}\right)$.

17 A noter que l'apparition de nouveaux organes d'enroulement du câble élargit la gamme des équipements disponibles sans signifier un renoncement aux systèmes antérieurs qui ont continué d'évoluer en s'améliorant. Il suffit, pour s'en convaincre, de relever le nombre de poulies Koepe dans les deux bassins où elles équipent, depuis la fin du XIXe siècle, aussi bien les puits de service (poulie monocâble) que les puits d'extraction (poulies monocâbles, bicâbles ou quadricâbles) (fig. $\mathbf{n}^{\circ}$ 9). 
Figure 8

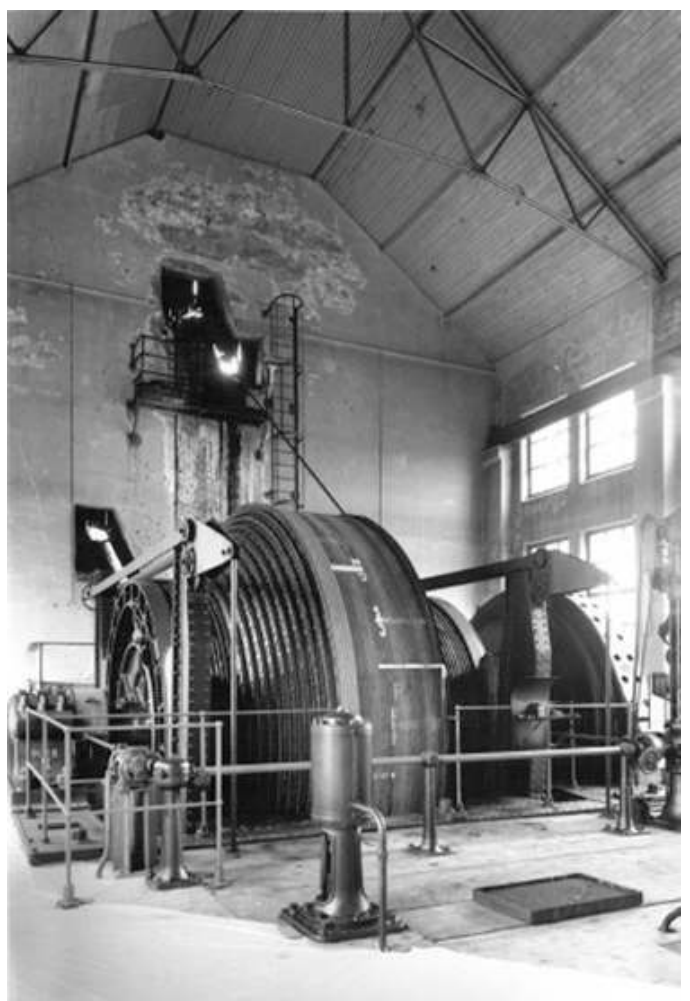

Tambour bicylindroconique de la machine d'extraction du puits Else, mise en route en 1929 (carreau Joseph-Else, Wittelsheim). Couturier, Bernard

(c) Inventaire général, ADAGP, 1994. 
Figure 9

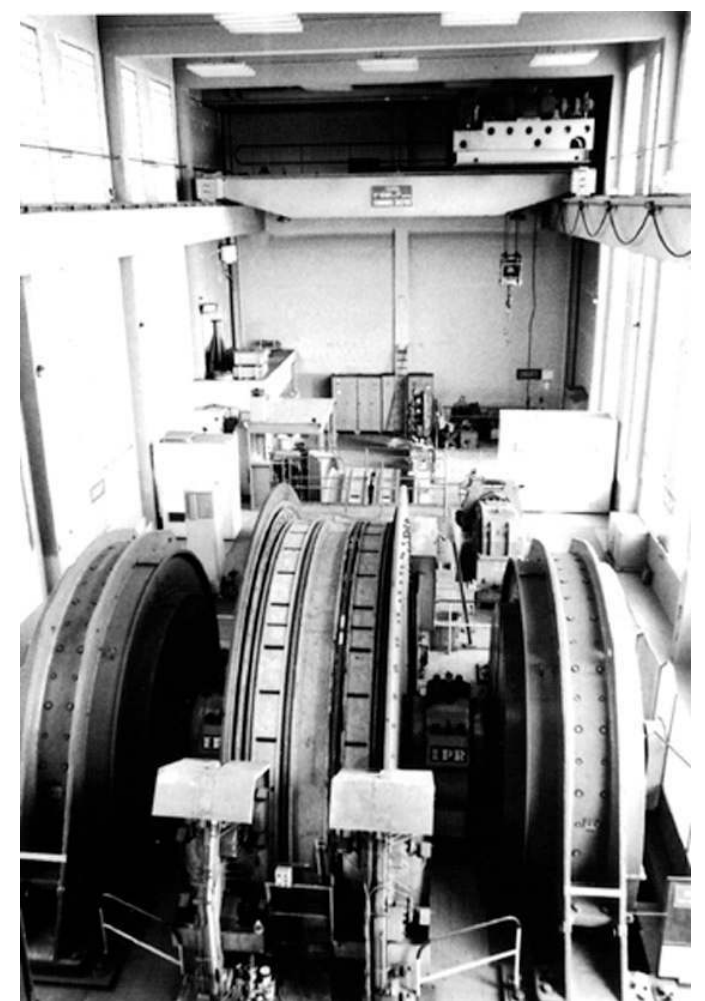

Poulie Koepe du puits Simon 5 (tour d'extraction, Forbach). Buchheit, Chip (c) Buchheit, Chip,1997.

Tableau B : relevé des tambours, bobines et poulies (non compris les machines de secours

\begin{tabular}{|l|c|c|c|c|}
\hline & $\begin{array}{c}\text { bobine } \\
\text { (câble plat) }\end{array}$ & poulie Koepe & \multicolumn{2}{|c|}{ tambour } \\
\hline & & & cylindrique & bicylindroconique \\
\hline Bassin houiller & 2 & 16 & 1 & 1 \\
\hline Mines de potasse & $\left(1^{*}\right)$ & 8 & - & $4\left(2^{*}\right)$ \\
\hline
\end{tabular}

$\left(1^{*}\right)$ : la bobine signalée a été combinée à une poulie Koepe (puits Rodolphe 1, Pulversheim).

$(2 *)$ : dont 2 machines disparues après inventaire.

\section{Résultat des recensements}

La méthode adoptée pour évaluer le patrimoine minier ne diffère en rien de celle qu'on applique habituellement aux édifices et aux objets des autres secteurs de l'industrie. Elle s'appuie, notamment, sur une datation de toutes les installations et sur la recherche des “ensembles" ou, à défaut, des "éléments isolés" susceptibles de pouvoir représenter l'une ou l'autre des périodes délimitées?.

Rappelons qu'au total, 55 puits de mines furent foncés et équipés dans le bassin houiller lorrain $^{8}$ et 24 dans le bassin potassique haut-rhinois ${ }^{9}$. Mais le nombre des équipements 
installés au cours des temps est très supérieur à ces chiffres car certains puits ont connu jusqu'à 4 systèmes d'extraction successifs. Au moment où s'engageaient les recensements dans les deux bassins miniers (1996-1998 et 2003-2004) ${ }^{10}$, de nombreux puits avaient déjà totalement disparu avec tous leurs équipements et d'autres étaient soit partiellement démantelés, soit en passe de l'être. Ne restaient respectivement à étudier que 30 systèmes d'extraction (18 dans le bassin houiller et 12 dans le bassin potassique).

\section{Les systèmes d'extraction représentatifs et remarquables}

Au terme des études, 15 de ces systèmes en place dans le bassin houiller sont apparus représentatifs car leurs équipements, composés d'éléments contemporains, montraient la cohérence architecturale et technique recherchée. Ce résultat ne doit pas surprendre, car la plupart des puits considérés (dont 9 en activité au moment du recensement) ont été établis ou entièrement rééquipés au lendemain de la Seconde Guerre mondiale, sur fond de nationalisation (1946), de reconstruction et conformément aux plans de modernisation et de concentration industrielle voulus par le plan Monnet. Les houillères de Lorraine devaient tripler leur production en 10 ans.

\section{5 systèmes d'extraction représentatifs dans le bassin houiller lorrain, dont 4} remarquables qui ont été protégés (situation en 1998) ${ }^{11}$

- puits Vuillemin 2 (Petite-Rosselle) : système d'extraction 1882-1885 (protégé en 1998)

- puits Simon 1 (Forbach) : système d'extraction 1902 (protégé en 1998)

- puits Cuvelette Sud (Freyming-Merlebach) : système d'extraction 1930 (protégé en 1992)

- puits Wendel 2 (Petite-Rosselle) : système d'extraction 1947-1950

- puits Freyming (Freyming-Merlebach): système d'extraction 1949-1950

- puits Wendel 3 (Petite-Rosselle) : système d'extraction 1952-1954 (protégé en 1998) (fig. $\mathbf{n}$

- 10)

- puits Reumaux (Freyming-Merlebach) : système d'extraction 1953 (disparu)

- puits 4 La Houve (Creutzwald) : système d'extraction 1951-1955 (disparu)

- puits Simon 2 (Forbach) : système d'extraction 1955-1956

- puits Vouters (Freyming-Merlebach) : système d'extraction 1962

- puits Simon 5 (Forbach) : système d'extraction 1964-1966

- puits 3 La Houve (Creutzwald) : système d'extraction, 1982-1983 (disparu)

- puits De Vernejoul (Porcelette) : système d'extraction 1983 (disparu)

- puits Ouest (Diesen) : système d'extraction 1988 (disparu)

- puits Marienau (Forbach) : système d'extraction 1961-1962 (disparu) 
Figure 10

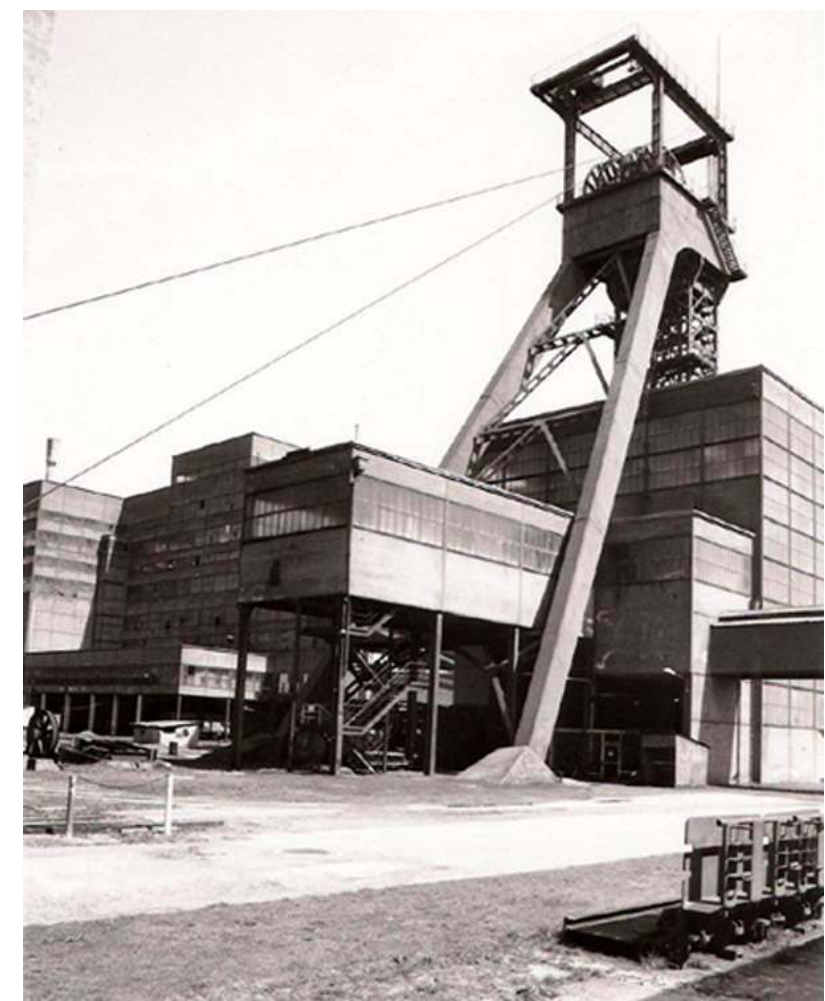

Le système d'extraction du puits Wendel 3 (Petite-Rosselle), mis en route en 1954 et aujourd'hui protégé. CCSTI-Musée du bassin houiller lorrain

(c) CCSTI-Musée du bassin houiller lorrain.

Le résultat apparait moins positif dans le bassin potassique, avec 4 systèmes d'extraction représentatifs sur 12, alors que les années 1946-1973 correspondent également à une période de grande expansion et de concentration industrielle. Ici, la réorganisation née de la fusion des concessions MDPA et Kali Sainte-Thérèse, entre 1945 et 1957, apparaît moins radicale. Elle s'est accommodée du renforcement partiel de systèmes d'extraction existants, voire de l'association d'équipements anciens et d'équipements neufs, comme au puits de Berrwiller par exemple, qui fut équipé lors de sa mise en route, en 1961, d'une machine provenant du puits Marie-Louise (Staffelfelden), dûment révisée mais datant de 1912.

\section{4 systèmes d'extraction représentatifs dans le bassin potassique alsacien, dont 1 a été protégé (situation en 2004)}

- puits Rodolphe 1 (Pulversheim) : système d'extraction 1910-1913-1929 ${ }^{12}$ (fig. $\mathbf{n}^{\circ} 11$ )

- puits Rodolphe 2 (Pulversheim) : système d'extraction 1926-1928 ${ }^{13}$

- puits Théodore (Wittenheim) : système d'extraction 1958-1968 ${ }^{14}$ (protégé en 1995)

- puits Staffelfelden (Staffelfelden) : système d'extraction 1971-1972 ${ }^{15}$ (disparu). 
Figure 11

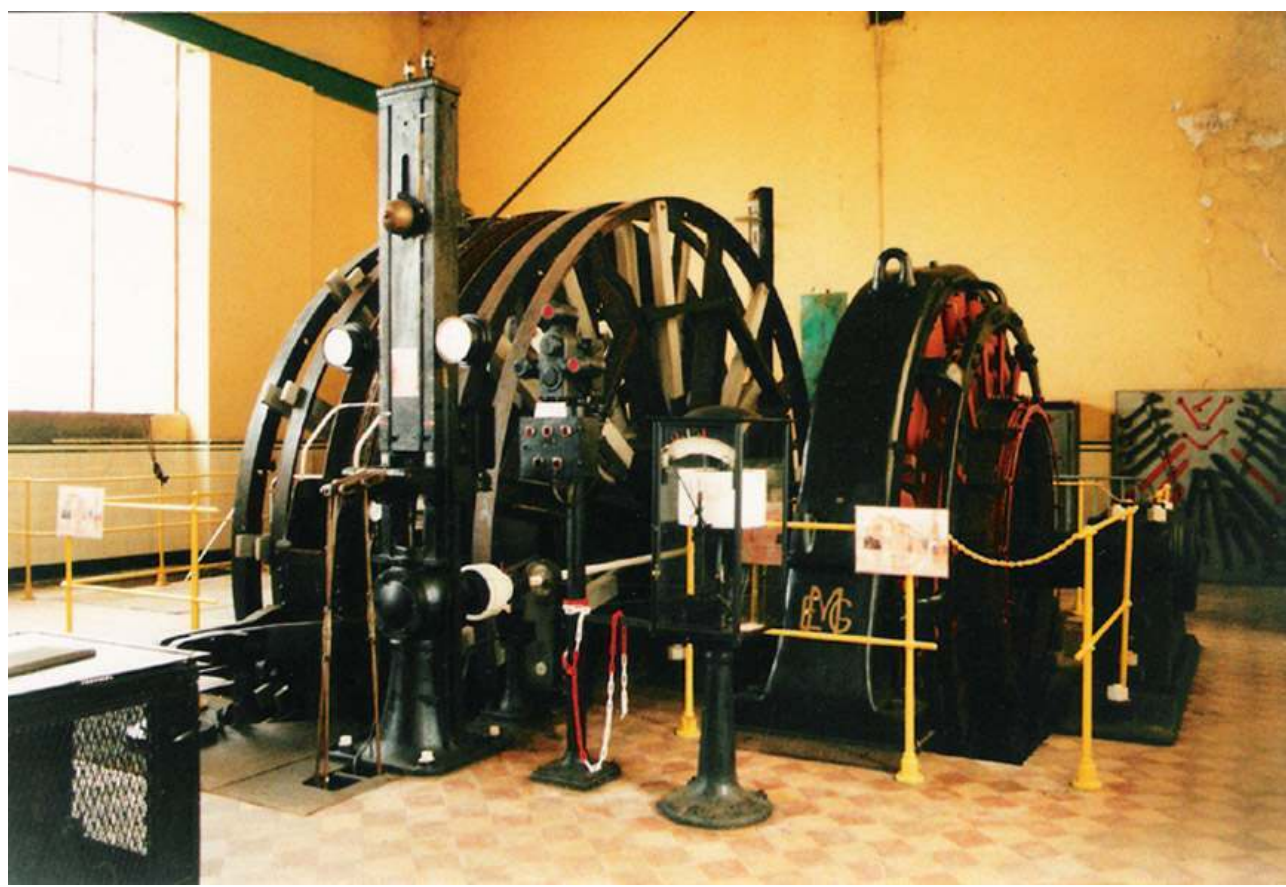

Machine d'extraction du puits Rodolphe 1 combinant une bobine et une poulie Koepe. Buchheit, Chip (c) Buchheit, Chip, 2003.

Il faut noter que ces renouvellements partiels répondaient parfaitement aux besoins des puits de service, mais ils ont perturbé la cohérence technique du "groupe extraction". Plusieurs puits présentant des équipements intéressants par leurs caractéristiques techniques, leur ancienneté ou leur rareté n'ont pas pu être signalés comme tels parce qu'intégrés à des systèmes d'extraction " composites".

La situation s'est rencontrée à Berrwiller ${ }^{16}$, on l'a dit, mais aussi à Richwiller (puits Max ${ }^{17}$ ) (fig. $\mathbf{n}^{\circ} 12$ ) et à Wittelsheim (puits Amélie $1^{18}$; puits Amélie $2^{19}$; puits Else ${ }^{20} ;$ puits Joseph ${ }^{21}$ ). La machine de ce dernier puits, en particulier, présente un volant d'inertie caréné qui n'a, croit-on, aucun équivalent. 


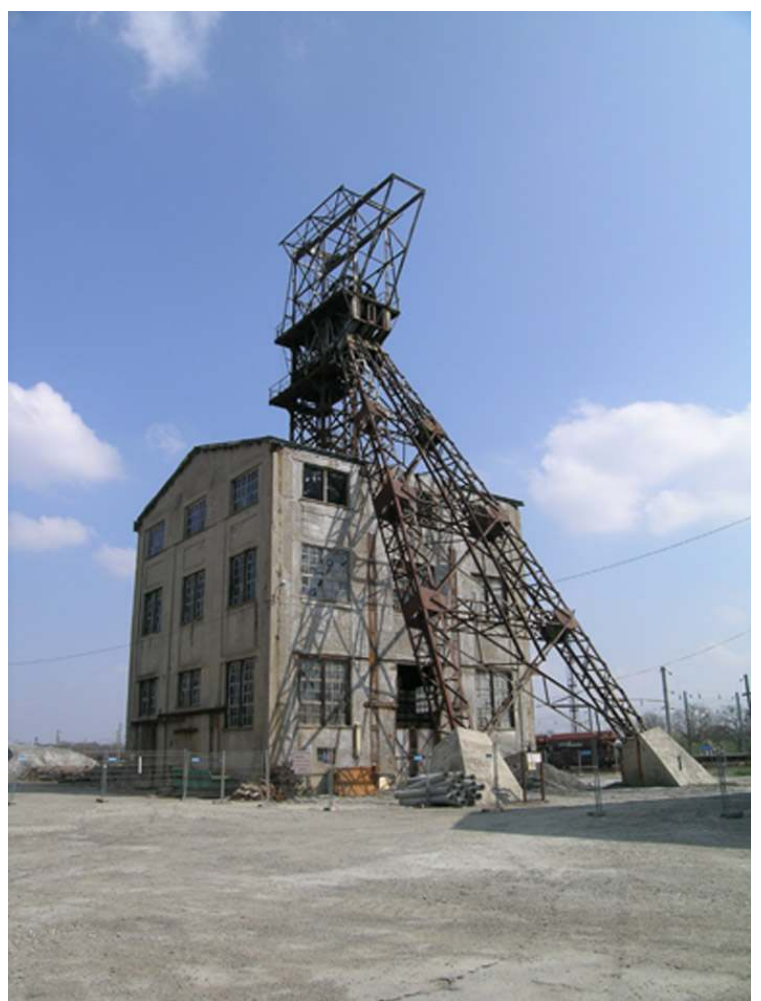

Un système d'extraction perturbé au puits Max (Richwiller). Le chevalement et la machine remontaient à 1911-1912, la recette à 1923 et le second moteur d'extraction à 1930. Buchheit, Chip (c) Buchheit, Chip, 2003

\section{Au panthéon de la mine}

Dûment recensées et documentées dans le cadre d'études thématiques, la trentaine de machines d'extraction étudiées constituaient bien une collection technique d'exception, non seulement pour les spécificités techniques remarquables qu'elles pouvaient présenter, mais aussi parce que tributaires d'une activité réputée définitivement révolue. En aucun lieu on n'a pourtant vu de machine d'extraction sauvegardée pour son seul intérêt. Pourquoi? Parce qu'élément d'un système technique indissociable? Pas seulement, car on peut observer aussi que les machines d'extraction, dans les ouvrages méthodologiques de l'Inventaire général, ont rang de "convertisseurs de couple "22, et que cette manière de voir a peut-être empêché de les considérer comme des machines à part entière, susceptibles d'être sauvegardées en tant que telles. En aurait-il été de même si elles avaient été rangées dans la catégorie des "machines à lever à bâti fixe", ce qu'elles sont aussi?

La raison principale relève, d'évidence, d'une tout autre dimension, car si une quinzaine de chevalements ou de tours d'extraction ont été préservés en France, en "isolé " et en dépit de leur total dépouillement, c'est bien parce que la silhouette de ces drôles de "charpentes à molettes" a été investie d'une dimension symbolique proprement monumentale. La machine d'extraction ne figure pas au panthéon. 


\section{NOTES}

1. Les modes d'exploitation "de surface" recouvrent le "grattage", le "grappillage" ou le " jardinage" des affleurements ainsi que tous les modes d'exploitation par "fendues" (galeries creusées dans le flanc des collines).

2. DAUMAS, Maurice (dir.). Histoire Générale des techniques. Paris : PUF, vol. 3, chap. II, p. 72.

3. C'est en 1902 que sont présentées les premières machines d'extraction électriques (Exposition minière de Düsseldorf) tandis que les dernières installations de machines marchant à la vapeur datent de 1954 (Ruhr).

4. Option préconisée par la Commission technique des houillères sinistrées, adoptée par le Gouvernement français lorsqu'il fallut reconstituer, entre 1919 et 1926, le potentiel des mines sinistrées du Nord et du Pas-de-Calais. BUCHHEIT, Chip. Histoire parallèle : machines et machinistes d'extraction dans le bassin houiller lorrain. CCSTI - Musée du bassin houiller lorrain avec le soutien de la DRAC-Lorraine. Non publié, 1994 (175 p.).

5. Les puits secondaires peuvent assurer différents services (mais pas d'extraction), à savoir: l'aérage des chantiers du fond, l'épuisement des eaux, la translation du personnel et du matériel, la descente de remblais.

6. Le système à " câble d'équilibre et poulie d'adhérence" fut conçu par deux ingénieurs : le Français Lemielle, en 1858 (invention sans suite) et l'Allemand Koepe, en 1878, qui en industrialisa le procédé.

7. On considère qu'un "ensemble" ou un "élément isolé" est représentatif quand il est fonctionnellement complet et que tous ses éléments sont contemporains (gages de cohérence architecturale et technique). On qualifie de "remarquables", les équipements représentatifs qui sont en outre rares (une rareté mesurée autant que possible à deux échelles : aire d'étude et territoire national).

8. Auxquels se sont ajoutés les puits Saint-Charles 4 et Merlebach Nord, implantés en Sarre et mis en exploitation en 1952 et 1953 pour exploiter le gisement sarrois.

9. Non comptés les puits Hettenschlag, Blodelsheim et Amélie 3, qui n’ont pas abouti.

10. Etude des installations minières de surface du bassin houiller lorrain (pour la DRACLorraine, 1996-1998) et Etude des installations minières de surface dans le bassin potassique haut-rhinois (DRAC-Alsace, Service de l'Inventaire, 2003-2004).

11. L'étude réalisée en plusieurs tranches, en 1994 et 1996-1998, fait actuellement l'objet de compléments photographiques et d'une extension prenant en compte l'ensemble du bâti lié à l'exploitation des houillères. Les notices seront accessibles sur les bases de données nationales en 2008. Les dossiers en cours sont consultables sur rendez-vous au Conseil régional de Lorraine, service régional de l'Inventaire général du patrimoine culturel, 29 rue du Haut-Bourgeois, 54000 Nancy.

12. Voir dans la base Palissy : notice IM68008686.

13. Voir dans la base Palissy : notice IM68008687.

14. Voir dans la base Palissy : notice IM68008682.

15. Voir dans la base Palissy : notice IM68008679.

16. Voir dans la base Palissy : notice IM68008683.

17. Voir dans la base Palissy : notice IM68008678.

18. Voir dans la base Palissy : notice IM68008680.

19. Voir dans la base Palissy : notice IM68008681.

20. Voir dans la base Palissy : notice IM68008689. 
21. Voir dans la base Palissy : notice IM68008688.

22. Thesaurus des Objets mobiliers. Paris : Editions du Patrimoine, 2001, p. 46 (coll. Documents \& Méthodes, $\left.n^{\circ} 8\right)$.

\section{RÉSUMÉS}

Cet article dresse un bref historique des machines d'extraction et rappelle les grandes étapes de leur évolution technique. Les résultats des recensements du patrimoine minier réalisés dans le bassin houiller lorrain et dans le bassin potassique alsacien ont permis de distinguer les éléments représentatifs et remarquables de ce patrimoine méconnu et insuffisamment considéré.

This article offers a brief history of winding engines and of the principal stages in their technical evolution. Inventory surveys carried out in the Lorraine coal basin and in the region of potassium mines in Alsace allow for an identification of the representative engines and of the remarkable ones, all of them part of a little studied and much neglected industrial heritage.

\section{INDEX}

Keywords : Alsace, coal mines, mining, Moselle, potassium mines, winding engines, Winding engines

Mots-clés : Alsace, industrie extractive, machine d'extraction, mine de houille, mine de potasse, Moselle

\section{AUTEUR}

\section{CHIP BUCHHEIT}

Chercheur. Chargée d'études pour le patrimoine industriel auprès du Service chargé de l'inventaire général du patrimoine culturel, Région Alsace. bielle.manivelle@wanadoo.fr 